\title{
Association between Latent Trajectories of Fluid Balance and Clinical Outcomes in Critically III Patients with Acute Kidney Injury: A Prospective Multicenter Observational Study
}

\author{
Meiping Wanga,b Bo Zhu ${ }^{a}$ Li Jiang ${ }^{a, c}$ Xuying Luo ${ }^{d}$ Na Wang ${ }^{e}$ Yibing Zhu ${ }^{a, f}$ \\ Xiuming $\mathrm{Xi}^{\mathrm{a}}$ Beijing Acute Kidney Injury Trial (BAKIT) Workgroup \\ ${ }^{a}$ Department of Critical Care Medicine, Fuxing Hospital, Capital Medical University, Beijing, China; ${ }^{b}$ Department of \\ Epidemiology and Health Statistics, School of Public Health, Capital Medical University, Beijing, China; 'Department \\ of Critical Care Medicine, Xuanwu Hospital, Capital Medical University, Beijing, China; ${ }^{d}$ Department of Critical Care \\ Medicine, Tiantan Hospital, Capital Medical University, Beijing, China; eEmergency Department, China Rehabilitation \\ Research Center, Capital Medical University, Beijing, China; ${ }^{f}$ Medical Research and Biometrics Center, Fuwai Hospital, \\ National Center for Cardiovascular Diseases, Peking Union Medical College, Chinese Academy of Medical Sciences, \\ Beijing, China
}

\section{Keywords}

Trajectory · Fluid balance $\cdot$ Fluid overload $\cdot$ Mortality $\cdot$ Acute kidney injury

\begin{abstract}
Introduction: We aimed to identify different trajectories of fluid balance (FB) and investigate the effect of FB trajectories on clinical outcomes in intensive care unit (ICU) patients with acute kidney injury (AKI) and the dose-response association between fluid overload (FO) and mortality. Methods: We derived data from the Beijing Acute Kidney Injury Trial (BAKIT). A total of 1,529 critically ill patients with AKI were included. The primary outcome was 28-day mortality, and hospital mortality, ICU mortality and AKI stage were the secondary outcomes. A group-based trajectory model was used to identify the trajectory of FB during the first 7 days. Multivariable logistic regression was performed to examine the relationship between FB trajectories and clinical outcomes. A lo-
\end{abstract}

gistic regression model with restricted cubic splines was used to examine the dose relationship between FO and 28day mortality. Results: Three distinct trajectories of FB were identified: low FB (1,316, 86.1\%), decreasing FB (120, 7.8\%), and high FB $(93,6.1 \%)$. Compared with low FB, high FB was associated with increased 28-day mortality (odds ratio [OR] 1.94, 95\% confidence interval [CI 1.17-3.19) and AKI stage (OR 2.04, 95\% Cl 1.23-3.37), whereas decreasing FB was associated with a reduction in 28-day mortality by approximately half (OR $0.53,95 \% \mathrm{Cl} 0.32-0.87$ ). Similar results were found for the outcomes of ICU mortality and hospital mortality. We observed a J-shaped relationship between maximum FO and 28-day mortality, with the lowest risk at a maximum FO of $2.8 \% \mathrm{~L} / \mathrm{kg}$. Conclusion: Different trajectories of FB in critically ill patients with AKI were associated with clinical

A complete list of the Beijing Acute Kidney Injury Trial (BAKIT) workgroup can be found in the Acknowledgements section. Meiping Wang and Bo Zhu contributed equally to this work. karger@karger.com www.karger.com/kdd

Karger $\frac{1}{\%}$

BOPEN ACCESS
(C) 2021 The Author(s)

Published by S. Karger AG, Basel

This is an Open Access article licensed under the Creative Commons Attribution-NonCommercial-4.0 International License (CC BY-NC) (http://www.karger.com/Services/OpenAccessLicense), applicable to the online version of the article only. Usage and distribution for commercial purposes requires written permission.
Correspondence to:

Xiuming Xi, xixiuming2937@163.com 
outcomes. An FB above or below a certain range was associated with an increased risk of mortality. Further studies should explore this relationship and search for the optimal fluid management strategies for critically ill patients with AKI.

(c) 2021 The Author(s)

Published by S. Karger AG, Basel

\section{Introduction}

More than half of patients in the intensive care unit (ICU) experience acute kidney injury (AKI), which is well recognized to be associated with increased mortality, length of stay in the ICU, and health-care costs [1-3]. The aim of early resuscitation is to expand the intravascular volume, ensure hemodynamic stability, and improve outcomes $[4,5]$. However, this practice may increase the risk of fluid overload (FO).

Multiple studies have shown that FO is associated with an increased risk of mortality and AKI severity [2, 6-9]. Nevertheless, the definition of FO is still debated. Previous studies have defined FO as cumulative fluid exceeding $10 \%$ of body weight (BW) $[6,10-12]$ or $5 \%$ of BW [13] or any positive fluid balance (FB) [7]. All of those studies hypothesized a linear association between FO and outcomes, which indicates that a higher FB or FO is related to adverse clinical outcomes. However, there were also studies showing that both a positive $\mathrm{FB}$ and a negative $\mathrm{FB}$ were associated with adverse outcomes $[14,15]$. It remains unclear whether there is a "sweet spot" of FB or FO, and the dose-response relationship between FO and outcomes in critically ill patients with AKI is still unknown.

Furthermore, the use of a single arbitrary point to define FO may not provide enough information to explore the effect of FO on clinical outcomes. Recently, a prospective study used latent class growth modeling and found that the latent trajectory of FB was associated with outcomes in patients receiving cardiac or aortic surgery [16]. Yende and colleagues [17] showed that persistently elevated trajectories of inflammation were associated with an increased risk of 1-year mortality and readmission. However, it remains unclear whether there are similar trajectories of FB in critically ill patients with AKI, and the association between FB trajectories and clinical outcomes is also unknown. Based on the Beijing Acute Kidney Injury Trial (BAKIT), the aim of our study was to identify different trajectories of FB and investigate the effect of FB trajectories on clinical outcomes in ICU patients with AKI and the dose-response association between maximum FO and 28-day mortality.

Trajectories of Fluid Balance and Clinical Outcomes in AKI Patients

\section{Methods}

\section{Study Participants}

This study population was from the BAKIT, which was conducted between March 1 and August 31,2012. Details on the design and major results of the BAKIT have been described previously [1, $6,18]$. The BAKIT was a prospective, multicenter, observational study conducted at 30 ICUs of 28 tertiary hospitals in Beijing, China. Patients who were admitted consecutively to ICUs and expected to stay in ICUs for at least $24 \mathrm{~h}$ were included. For patients who were admitted to ICUs more than once, only the initial ICU admission was considered. Patients with a diagnosis of end-stage CKD or those who had undergone renal replacement therapy (RRT) or received kidney transplant in the previous 3 months were excluded.

\section{Data Collection}

Baseline data included demographic information, primary diagnosis, comorbidities, sources of admission, and daily vital signs. The severity of illness was defined by the Acute Physiology and Chronic Health Evaluation II (APACHE II) score [19], the Sequential Organ Failure Assessment (SOFA) score [20], and the Simplified Acute Physiology Score II (SAPS II) [21] at admission. Serum creatinine (SCr), urine output, fluid intake and output were also collected daily. The need for RRT, mechanical ventilation (MV), or drug usage was also reported. The patients were followed up for 28 days until death or discharge from the ICU, whichever occurred earlier.

\section{Clinical Outcomes}

The primary outcome was 28-day mortality. ICU/hospital mortality (which was defined as the status of patient survival before ICU/hospital discharge), AKI stage, and length of stay in the ICU and hospital were secondary outcomes.

\section{Definitions}

The Kidney Disease Improving Global Outcomes (KDIGO) classification was used to diagnose AKI and the AKI stage [22]. The lowest SCr value during the previous 3 months was the baseline SCr. For patients with unknown values, the baseline SCr was estimated by using the Modification of Diet in Renal Disease (MDRD) equation [23] for an assumed glomerular filtration rate of $75 \mathrm{~mL} /$ $\mathrm{min} / 1.73 \mathrm{~m}^{2}[24,25]$ or the lowest SCr value during their ICU stay, whichever was lower [2]. In the BAKIT, the baseline SCr value was not known in 754 patients, the MDRD formula was used in 120 patients, and the lowest SCr values during the ICU stay were used in 634 patients to estimate the baseline SCr [1]. Patients were evaluated daily until day 10 or discharge from the ICU, and the worst stage during the patient's ICU stay was used.

Daily fluid intake included all intravenous and oral fluids, and daily fluid output included urine output, ultrafiltration fluid, drain fluid, and estimated gastrointestinal losses. Daily FB was defined as (fluid intake-fluid output, $\mathrm{mL}$ )/the initial BW (kg). FO was calculated by the ratio cumulative FB (liters, L) and the initial BW $(\mathrm{kg})$, and is reported as a percentage. Maximum FO was defined as the peak value of FO observed during a 7-day ICU stay.

\section{Statistical Analysis}

Categorical variables are expressed as the number of cases and proportions, and were compared by using the Mantel-Haenszel $\chi^{2}$ test. Non-normally distributed continuous variables are expressed as the median and interquartile range, and were compared using 
Table 1. Characteristics and clinical outcomes of the 3 subgroups with different FB trajectory patterns

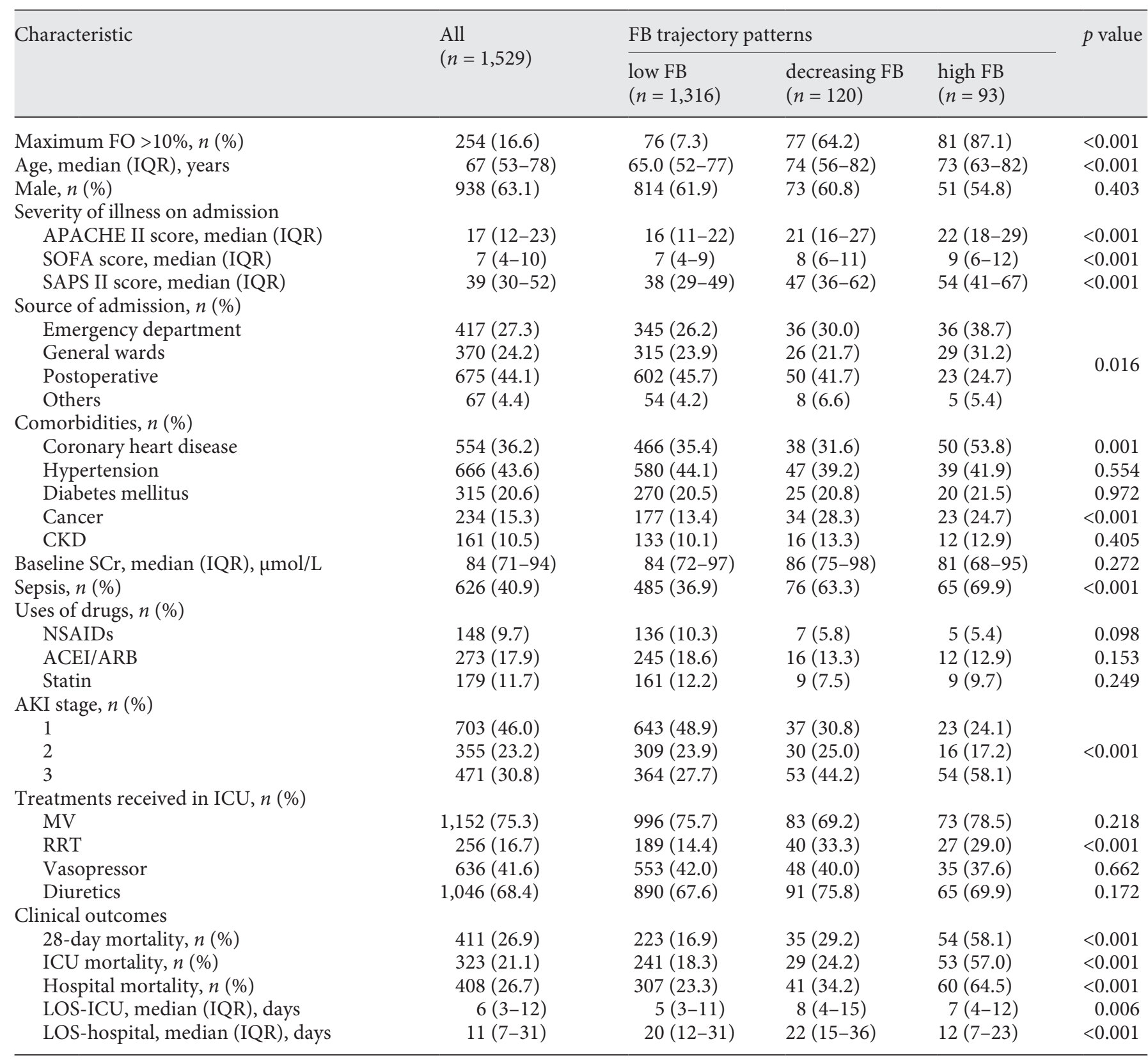

FB, fluid balance; IQR, interquartile range; APACHE II, Acute Physiology and Chronic Health Evaluation II; SAPS II, Simplified Acute Physiology Score II; SOFA, Sequential Organ Failure Assessment; SCr, serum creatinine; NSAIDs, nonsteroidal anti-inflammatory drugs; ACEI, angiotensin-converting enzyme inhibitor; ARB, angiotensin receptor blocker; ICU, intensive care unit; AKI, acute kidney injury; LOS, length of stay; FO, fluid overload; RRT, renal replacement therapy; MV, mechanical ventilation.

the Mann-Whitney U test or the Kruskal-Wallis analysis of variance test with Bonferroni correction.

To identify latent clusters of trajectories in the subjects following a similar process of change over time for $\mathrm{FB}$, we conducted a groupbased trajectory model (GBTM), which is a semiparametric statistical approach and assumes that the subjects are heterogeneous and composed a finite number of distinct groups [26]. We adopted the
Bayesian information criterion (BIC) to identify optimal numbers of subgroups and appropriate orders of heterogeneity (linear, quadratic, or cubic) in trajectories [26, 27]. A lower BIC value represents a better model fit. Furthermore, the sample size should include at least $5 \%$ of subjects, and the appropriate probability of each trajectory group was defined as $\geq 0.70[28,29]$. A censored normal distribution model was conducted using the traj plugin in Stata to estimate the
Wang et al. 
Fig. 1. FB trajectories for the 3 different clusters during the first 7 days after ICU admission. FB, fluid balance; ICU, intensive care unit.

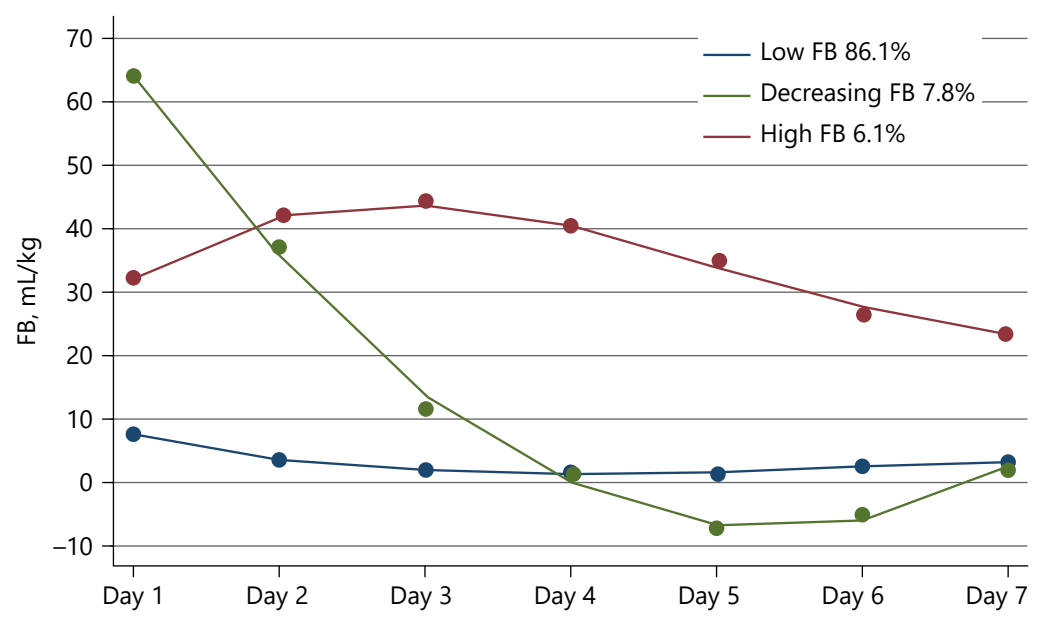

trajectory of FB in patients with AKI [30]. In this study, the BIC values from 2 clusters to 5 clusters were $-36,112.29,-35,866.9,-35,950.1$, and $-35,885$. Although a model with 5 trajectories had a lower BIC value, the number of patients in this model was $<5 \%$. Therefore, 3 distinct trajectories of daily FB were identified in the following analyses. Boxplots were used to illustrate the daily FB and cumulative FB in latent trajectory groups during the first 7 days of ICU admission.

The association between latent trajectory subgroups and outcomes was tested by using univariate and multivariable logistic regression or ordered logistic regression analysis. In the multivariable model, we included age and sex as well as the APACHE II, SAPS II, and SOFA scores on admission, comorbidities, sepsis, therapy in the ICU, and maximum FO. The centers were also included in the multivariable models as a random effect. Unadjusted and adjusted odds ratios (aORs) and their 95\% confidence intervals (CIs) were calculated. To further evaluate the pattern and magnitude association between maximum FO and the primary outcome, we used a logistic regression model with restricted cubic splines (RCS) for FO adjusted for covariates [31]. A median maximum FO of $3.3 \% \mathrm{~L} / \mathrm{kg}$ was treated as the reference, and the 4 knots for the splines were placed at the 5 th, 35th, 65 th, and 95th percentiles of maximum FO.

In the sensitivity analysis, we excluded patients without baseline SCr values to exclude the potential influence of baseline kidney function on the effects of the AKI definition. We further explored the potential interaction between $\mathrm{FB}$ trajectories and the severity of illness (APACHE II $\geq$ vs. APACHE II $<20$ ), and sepsis (yes vs. no). Analyses were performed using Stata version 15 (Stata Corp, College Station, TX, USA). A $p$ value $<0.05$ was considered statistically significant.

\section{Results}

\section{Participants and Baseline Characteristics}

A total of 3,107 eligible patients were enrolled in the BAKIT. After excluding 1,523 patients without AKI and 55 patients with missing fluid intake/output data during the ICU stay, 1,529 patients were included in the final analysis (see online suppl. Fig. 1; for all online suppl. material, see www.karger.com/doi/10.1159/000515533). The baseline clinical characteristics and outcomes are shown in Table 1. Among the 1,529 patients, the median age was $67(53-78)$ years, and 938 patients (61.3\%) were male. The median APACHE II, SOFA, and SAPS II scores on admission to the ICU were 17 (12-23), 7 (4-10), and 39 (30-52), respectively. Patients with AKI more commonly received MV $(1,152,75.3 \%)$ and diuretics $(1,046$, $68.4 \%)$ than patients without AKI. The 28-day mortality rate was $26.9 \%$, and the median length of stay in the ICU was 6 days.

\section{Baseline Characteristics among the Three Latent Trajectory Groups}

Among 1,529 patients, 3 distinct trajectories were identified as subgroups with low, decreasing and high FB over 7 days (Fig. 1). Trajectory 1, described as "low FB" $(1,316,86.1 \%)$, showed subjects who maintained a low FB throughout the following 7 days. Trajectory 2, described as "decreasing FB" $(120,7.8 \%)$, represented a trend in which the subjects started with a high FB that decreased quickly from day 2 to day 4 and maintained a lower level. Trajectory 3, described as "high FB" (93, 6.1\%), showed a trend in which the subjects maintained a high FB from day 1 to day 5 and then slightly decreased. Even though we excluded the patients without baseline SCr values, similar distinct trajectories of FB still existed (online suppl. Fig. 2). The other 2 groups were more likely to be older, had higher severity illness scores on admission, received more RRT, and had poorer clinical outcomes than low FB patients (Table 1). 

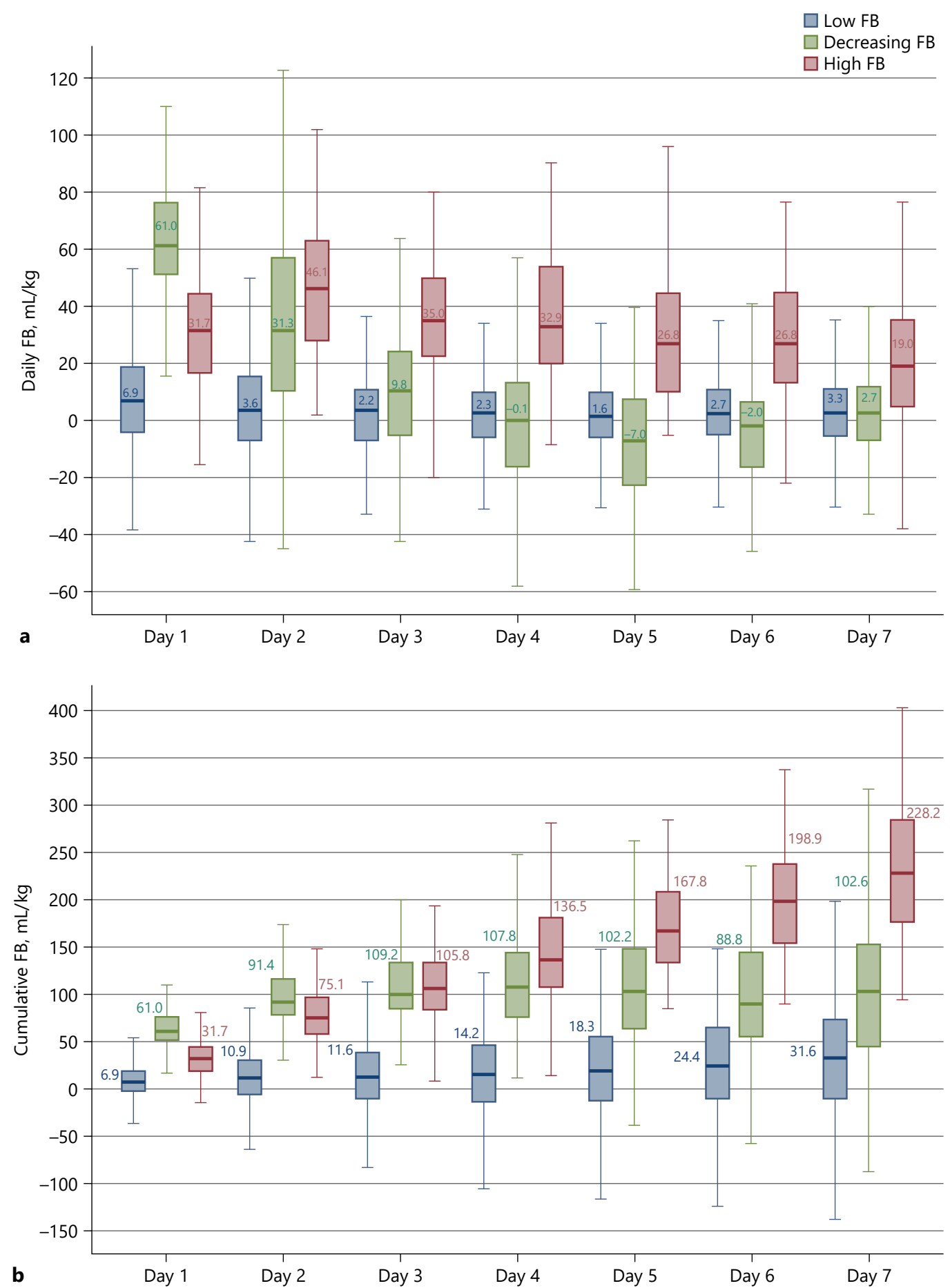

Fig. 2. Daily FB (a) and cumulative FB (b) by trajectory pattern. FB, fluid balance. 
Table 2. ORs for clinical outcomes among AKI patients with 3 different FB trajectory patterns

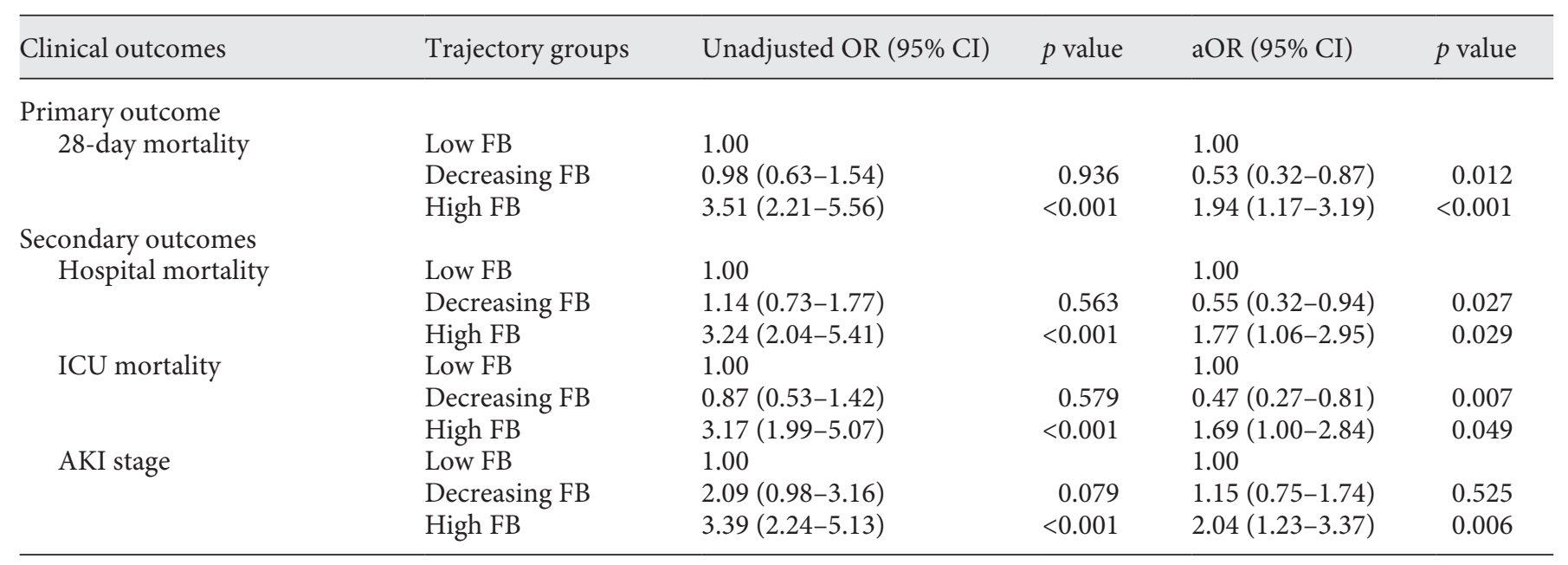

FB, fluid balance; ICU, intensive care unit; AKI, acute kidney injury; OR, odds ratio; CI, confidence interval; aOR, adjusted OR.

\section{FB in the Three Trajectory Clusters}

The 3 latent trajectory clusters showed significantly different degrees of daily FB at all time points. The decreasing FB cluster had the highest daily FB $(61.0 \mathrm{~mL} / \mathrm{kg})$ on the first day of ICU admission and then decreased rapidly to $31.3 \mathrm{~mL} / \mathrm{kg}$ on the second day. The low FB cluster had a slight change from 6.9 to $1.6 \mathrm{~mL} / \mathrm{kg}$ during their stay in the ICU. The high FB cluster had the highest FB of 46.5 $\mathrm{mL} / \mathrm{kg}$ on day 2 but remained at a relatively high level (19.0-35.0 mL/kg) during the following days (Fig. 2a).

There was a gradual increase in fluid accumulation in the low FB cluster (from 6.9 to $31.6 \mathrm{~mL} / \mathrm{kg}$ ) and a rapid increase in the high FB cluster (from 31.7 to $228.2 \mathrm{~mL} / \mathrm{kg}$ ) during the first 7 days of ICU admission. However, the decreasing FB cluster had a progressive cumulative FB on the first 3 days (from 61.0 to $109.2 \mathrm{~mL} / \mathrm{kg}$ ) of admission and then had a rapidly decreasing trend (Fig. 2b).

\section{ORs for the Association between the Three Latent \\ Clusters and Clinical Outcomes}

The ORs of clinical outcomes based on the trajectories of FB are shown in Table 2. Compared with the low FB cluster, the decreasing FB cluster had an approximately one-half decreased risk of 28-day mortality after adjusting for potential confounders (aOR 0.53, 95\% CI 0.32-0.87), whereas the high FB cluster had an approximately 2.0 -fold increased risk of 28-day mortality after adjusting for potential confounders (aOR 1.94, 95\% CI 1.17-3.19). Similar results were found for the outcomes of ICU mortality and hospital mortality. When ordered logistic regression was used to assess the association between the AKI stage and latent trajectories of $\mathrm{FB}$, we found that the decreasing $\mathrm{FB}$ cluster showed a trend of an increased risk of severe AKI although the difference was not statistically significant (aOR 1.15, 95\% CI 0.75-1.74). However, the cluster with high FB had an approximately 2.0 -fold increased risk of severe AKI (aOR 2.04, 95\% CI 1.23-3.37). A similar association between the trajectories of FB and clinical outcomes was found after excluding those without baseline SCr values (online suppl. Table 1). However, there were significant interactions between the trajectories of FB and 28 -day mortality. The association between the trajectories of FB and 28-day mortality was stronger in patients with sepsis (decreasing FB, OR [95\% CI]: 0.65 [0.37-1.15]; high FB, OR [95\% CI]: 1.69 [1.04-3.16]) than in those without sepsis (decreasing FB, OR [95\% CI]: 0.82 [0.56-1.58]; high FB, OR [ $95 \%$ CI]: 1.27 [1.00-1.62]), and the $p$ value for the interaction was 0.032 (online suppl. Table 2).

\section{Association between FO and 28-Day Mortality}

From day 1 to day 7, the proportion of patients with FO increased 24.0 -fold (0.9\% day $1-21.9 \%$ day 7 ) (online suppl. Fig. 3). Compared with the low FB cluster, the other 2 clusters had a significantly increased maximum FO (7.3 vs. 64.2 vs. $87.1 \%$, Table 1 ). The maximum FO had a skewed distribution, with a median of $3.3(0.6-7.8) \% \mathrm{~L} /$ $\mathrm{kg}$ (online suppl. Fig. 4).

We also used a logistic regression model with an RCS model to estimate the trend in the risk of FO and 28-day mortality. The spline function for maximum FO demon- 

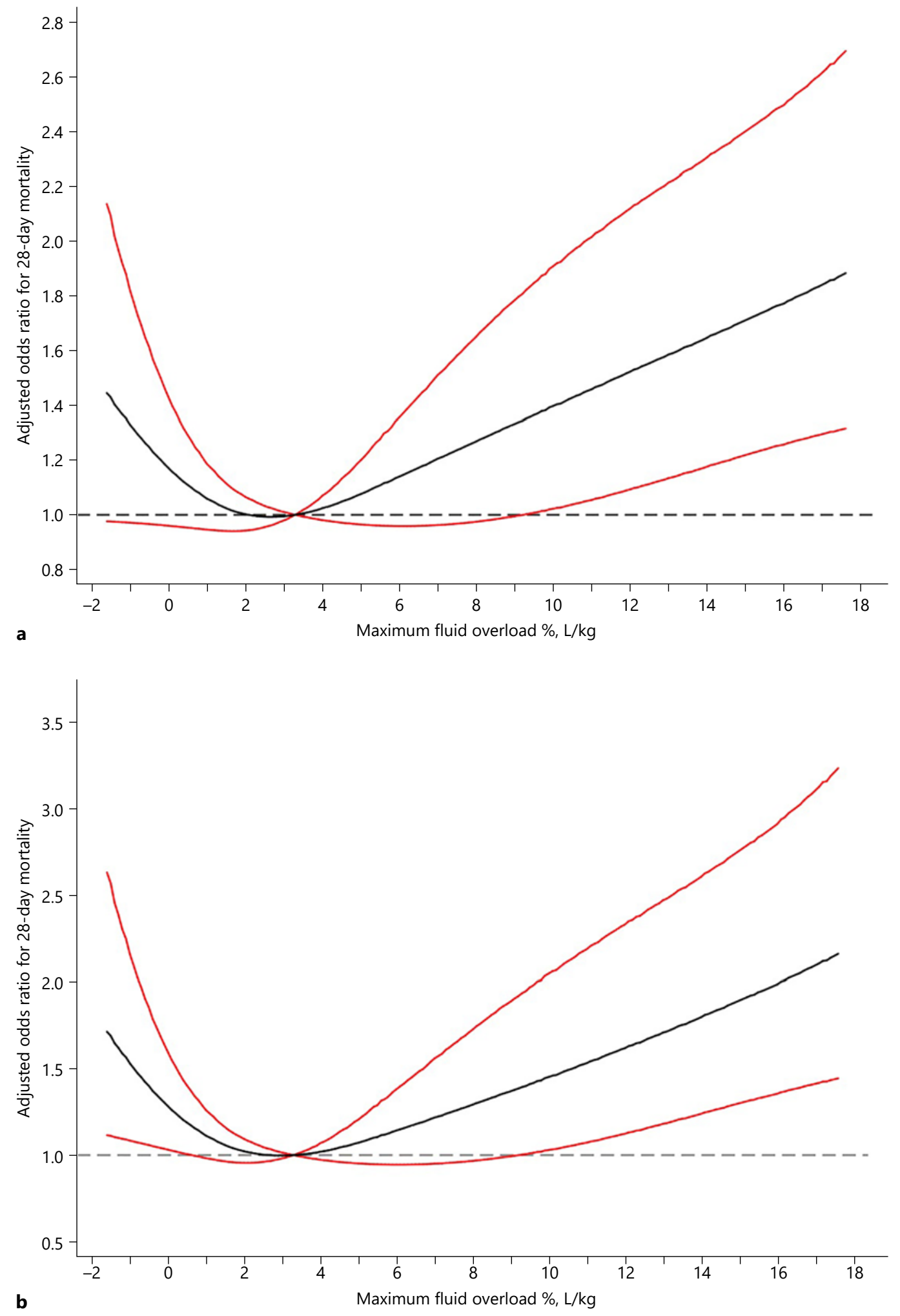

3

(For legend see next page.) 
strated a nonlinear, J-shaped association between maximum FO and 28-day mortality with the lowest risk at a maximum FO of $2.8 \% \mathrm{~L} / \mathrm{kg}$ (Fig. 3; online suppl. Fig. 5).

\section{Discussion}

In this prospective multicenter observational study, we identified 3 latent trajectory groups of $\mathrm{FB}$, characterized by low FB, decreasing $\mathrm{FB}$, and high $\mathrm{FB}$, in patients with AKI and found that the trajectories of FB were associated with 28 -day mortality. The individuals with a decreasing FB had a lower risk of 28 -day mortality, ICU mortality, and hospital mortality, but those with a high FB had an increased risk of mortality and severe AKI. Moreover, we demonstrated a J-shaped relationship between maximum FO and 28-day mortality, with the lowest risk at a maximum $\mathrm{FO}$ of $2.8 \% \mathrm{~L} / \mathrm{kg}$ from RCS analysis.

Fluid administration is an integral component of the management of critically ill patients. Although several strategies have been developed to estimate fluid responsiveness [32], optimal fluid management is still controversial. Early fluid resuscitation has been shown to reverse tissue hypoperfusion and improve patient outcomes $[4,33,34]$. The guidelines of the Surviving Sepsis Campaign recommend at least $30 \mathrm{~mL} / \mathrm{kg}$ intravenous crystalloid fluid within the first $3 \mathrm{~h}$ of onset of sepsis-induced tissue hypotension or sepsis [5]. Due to the lack of effective fluid responsiveness indicators, it could easily lead to positive post-resuscitation FB or FO along with its detrimental effects, such as increasing and prolonging the duration of multiorgan dysfunction or failure, increasing the risk of mortality, prolonging the need for MV, and increasing health-care costs [35-39].

Several studies have demonstrated that a positive FB or FO is associated with adverse outcomes in critically ill patients with AKI $[7,8,36,40]$. Raimundo and colleagues [41] indicated that for every $1-\mathrm{L}$ increase in FB, the risk of AKI progression increased 6-fold (OR 6.09, 95\% CI

Fig. 3. Relationship between maximum FO and aOR of 28-day mortality in critically ill patients with AKI. a All participants. b Excluding participants without baseline SCr values. The black line indicates the aOR, and the red lines indicate the 95\% CI bands. Data were fitted using a multivariate logistic regression model with RCS with 4 knots (5th, 35th, 65th, and 95th percentiles) for maximum FO adjusted for potential confounders. Due to the small sample sizes, the lowest $5 \%$ and highest $5 \%$ of participants are not shown in the figures. AKI, acute kidney injury; FO, fluid overload; aOR, adjusted odds ratio; RCS, restricted cubic splines; SCr, serum creatinine.

Trajectories of Fluid Balance and Clinical Outcomes in AKI Patients
2.39-15.52). Recently, a retrospective study demonstrated that peak FO within 3 days was associated with ventilator-associated conditions and infection-related ventilator-associated compliance in AKI children with MV [42]. Our previous study showed that FO was significantly related to the incidence of AKI and AKI severity, and a high cumulative FB at day 3 was an independent risk factor for 28-day mortality [6].

However, a single arbitrary FB value may not provide adequate information to evaluate clinical outcomes in critically ill patients. Recently, a prospective study involving patients undergoing cardiac and aortic surgery found that the trajectory of FB was associated with the risk of AKI and dialysis [16]. Our study used a similar GBTM to explore the trend of FB in critically ill patients with $\mathrm{AKI}$ and showed that there were 3 latent trajectory clusters: low FB, decreasing FB, and high FB. The patients in the decreasing FB group had as high as $61 \mathrm{~mL} /$ $\mathrm{kg}$ on the first day of ICU admission and then rapidly decreased to $31 \mathrm{~mL} / \mathrm{kg}$ on the second day. The peak volume of cumulative $\mathrm{FB}$ was $109 \mathrm{~mL} / \mathrm{kg}$ on the third day. The low FB group maintained a lower FB status. The patients in the high FB group maintained a higher FB status above $30 \mathrm{~mL} / \mathrm{kg}$ during the first 4 days following admission, with a cumulative $\mathrm{FB}$ as high as $230 \mathrm{~mL} / \mathrm{kg}$ on day 7. Compared to patients with low FB, the other 2 clusters had an increased maximum FO (7.3 vs. 64.2 vs. $87.1 \%$ ), whereas the decreasing FB patients had lower 28-day mortality (aOR 0.53, 95\% CI 0.32-0.87), hospital mortality (aOR $0.55,95 \%$ CI $0.32-0.94$ ), and ICU mortality (aOR $0.47,95 \%$ CI $0.27-0.81$ ) although $>60 \%$ of the patients in the decreasing FB group developed FO. In contrast, patients with a high FB had significantly increased risk of 28-day mortality (aOR 1.94, 95\% CI 1.17-3.19), hospital mortality (aOR 1.77, 95\% CI 1.06-2.95), and ICU mortality (aOR 1.69, 95\% CI 1.00-2.84). Furthermore, a high FB increased the risk of AKI severity by 2.0fold (aOR 2.04, 95\% CI 2.04-3.37). However, the association between the trajectories of FB and 28-day mortality was stronger in patients with sepsis than in those without sepsis. The distinct trajectories of FB may be good indexes and preferably reflect the whole condition in patients with AKI. These findings seem to be logical and are consistent with those of previous studies in which earlier fluid resuscitation was found to improve hypovolemia and tissue or organ hypotension, which are related to a lower risk of adverse outcomes [43].

In addition, previous studies have demonstrated that cumulative fluid excess with a cutoff value was associated with outcomes $[6,7,13]$. However, this cutoff value re- 
mains controversial. Studies on the dose-response association between FO and outcomes are scarce. In our study, we used RCS to assess the association between maximum $\mathrm{FO}$ and 28-day mortality and found a J-shaped association between maximum FO and 28-day mortality, with the lowest risk at a maximum FO of $2.8 \% \mathrm{~L} / \mathrm{kg}$. This nonlinear association suggests that a high maximum FO is closely associated with an increased risk of 28-day mortality. Despite the relatively small sample size of patients with a maximum FO $<2.8 \%$, we also observed increased 28-day mortality. Woodward and colleagues [44] excluded patients with an $\mathrm{FO}<0$ and found a $\mathrm{U}$-shaped association between FO and major adverse renal outcomes in ICU patients with AKI requiring continuous RRT. These results may suggest that not only a greater FO but also a lower FO is potentially harmful, and similar results have been observed in other studies $[14,15]$. This phenomenon may be related to insufficient resuscitation or volume depletion, renal or other organ hypoperfusion, increased AKI-related events, and mortality. Further studies should be performed to indicate the optimal range of FB.

Our study was the first to explore the association between FB and clinical outcomes by combining a doseresponse and latent growth mixed model in a large ICU population. Nonetheless, our study had some limitations. First, this observational study could not explore the causeeffect relationship between FB and outcomes, and we could not adjust for all potential confounders. Second, we did not consider the fluid intakes and outputs before ICU admission or in the operating room, which may influence FB and outcomes. Third, the insensible losses were not considered, although they are not negligible in assessing the effect of FB or FO on outcomes. Finally, different types of fluid have different influences on fluid accumulation and outcomes $[45,46]$.

\section{Conclusion}

Our results demonstrated that 3 different trajectories of FB occurred in critically ill patients with AKI. A decreasing FB was associated with reduced 28-day mortality. Furthermore, there was a dose-response association between FO and mortality, which suggests that a cumulative $\mathrm{FB}$ above or below a certain range increases the risk of poor outcomes in patients with AKI. Further studies should be performed to explore the effect of cumulative $\mathrm{FB}$ and the trend of FB on clinical outcomes to determine optimal fluid management strategies.

\section{Acknowledgements}

We gratefully thank the Beijing Municipal Science and Technology Commission, a government fund used to improve health-care quality and data collection, for its financial support. We also acknowledge all the following members of the BAKIT workgroup who contributed data and samples and have made this work possible: Yuan $\mathrm{Xu}$ (Department of Critical Care Medicine, Beijing Tongren Hospital, Capital Medical University); Jianxin Zhou (Department of Critical Care Medicine, Beijing Tiantan Hospital, Capital Medical University); Ang Li (Department of Critical Care Medicine, Beijing Friendship Hospital, Capital Medical University); Jingyuan Liu (Department of Critical Care Medicine, Beijing Ditan Hospital, Capital Medical University); Wenxiong Li (Surgical Intensive Care Unit, Beijing Chaoyang Hospital, Capital Medical University); Wenjin Chen (Neurological Intensive Care Unit, Xuanwu Hospital, Capital Medical University); Jianguo Jia (Surgical Intensive Care Unit, Xuanwu Hospital, Capital Medical University); Xi Zhu (Department of Critical Care Medicine, Peking University Third Hospital); Penglin Ma (Department of Critical Care Medicine, the 309th Hospital of Chinese People's Liberation Army); Wei Chen (Department of Critical Care Medicine, Beijing Shijitan Hospital, Capital Medical University); Dongxin Wang (Department of Critical Care Medicine, Peking University First Hospital); Youzhong An (Department of Critical Care Medicine, Peking University People's Hospital); Qingyuan Zhan (Department of Critical Care Medicine, China-Japan Friendship Hospital); Gang Li (Department of Critical Care Medicine, China-Japan Friendship Hospital); Haitao Zhang (Surgical Intensive Care Unit, Fuwai Hospital, China Academy of Medical Science and Peking Union Medical College); Bo Ning (Department of Critical Care Medicine, Air Force General Hospital of Chinese People's Liberation Army); Zhongjie He (Department of Critical Care Medicine, the First Affiliated Hospital of General Hospital of People's Liberation Army); Zhicheng Zhang (Department of Critical Care Medicine, Navy General Hospital); Yaxiong Sun (Department of Critical Care Medicine, the Luhe Teaching Hospital of the Capital Medical University); Shijie Jia (Surgical Intensive Care Unit, Beijing Anzhen Hospital, Capital Medical University); Yalin Liu (Surgical Intensive Care Unit, Beijing Hospital); Rui Cheng (Department of Critical Care Medicine, General Hospital of Armed Police Forces); Qing Song (Department of Critical Care Medicine, the General Hospital of People's Liberation Army); Jinning Liu (Surgical Intensive Care Unit, Beijing YouAn Hospital, Capital Medical University); Yangong Chao (Department of Critical Care Medicine, Hua Xin Hospital, First Hospital of Tsinghua University); Huizhen Li (Department of Critical Care Medicine, Beijing Shunyi Hospital, China Medical University); Li Feng (Department of Critical Care Medicine, Beijing Geriatric Hospital); Ruochun Shi (Department of Critical Care Medicine, Beijing No. 6 Hospital); and Xiuming Xi, Li Jiang, Ying Wen, Bo Zhu, Meiping Wang, Qi Jiang, Yanyan Yin, Zhen Zhao, and Ran Lou (Department of Critical Care Medicine, Fuxing Hospital, Capital Medical University).

\section{Statement of Ethics}

This study was approved by the Institutional Review Board of Fu Xing Hospital (2010XM0501) and all other participating hospitals (online suppl. material). The Institutional Review Board specifically approved the informed consent waiver because of the anonymous and purely observational nature of this study. 


\section{Conflict of Interest Statement}

The authors declare that they have no competing interests.

\section{Funding Sources}

This study was supported by the Beijing Municipal Science and Technology Commission, a government fund used to improve health-care quality (D101100050010058). The funding source had no role in the study design, management, data collection, analysis and writing of manuscript, or the decision to submit it for publication.

\section{Author Contributions}

X.M.X. designed the BAKIT and secured funding. M.P.W. and X.M.X. had full access to all of the data in the study and take responsibility for the integrity of the data and accuracy of analysis presented in this study. M.P.W. and B.Z. calculated the statistics and wrote the manuscript. L.J. was involved in study design, acquisition of data, and helped revise the manuscript. X.Y.L., N.W., and Y.B.Z. contributed substantially to the study design, data analysis and interpretation, and the writing of the manuscript. All authors critically reviewed the manuscript and agreed with the final version and findings prior to submission.

\section{References}

1 Luo X, Jiang L, Du B, Wen Y, Wang M, Xi X, et al. A comparison of different diagnostic criteria of acute kidney injury in critically ill patients. Crit Care. 2014;18(4):R144

2 Hoste EA, Bagshaw SM, Bellomo R, Cely CM, Colman R, Cruz DN, et al. Epidemiology of acute kidney injury in critically ill patients: the multinational AKI-EPI study. Intensive Care Med. 2015;41(8):1411-23.

3 Malhotra R, Kashani KB, Macedo E, Kim J, Bouchard J, Wynn S, et al. A risk prediction score for acute kidney injury in the intensive care unit. Nephrol Dial Transplant. 2017; 32(5):814-22.

4 Rivers E, Nguyen B, Havstad S, Ressler J, Muzzin A, Knoblich B, et al. Early goal-directed therapy in the treatment of severe sepsis and septic shock. N Engl J Med. 2001;345(19): 1368-77.

5 Rhodes A, Evans LE, Alhazzani W, Levy MM, Antonelli M, Ferrer R, et al. Surviving sepsis campaign: international guidelines for management of sepsis and septic shock: 2016. Intensive Care Med. 2017;43(3):304-77.

6 Wang N, Jiang L, Zhu B, Wen Y, Xi XM; Beijing Acute Kidney Injury Trial Workgroup. Fluid balance and mortality in critically ill patients with acute kidney injury: a multicenter prospective epidemiological study. Crit Care. 2015; 19:371.

7 Garzotto F, Ostermann M, Martín-Langerwerf D, Sánchez-Sánchez M, Teng J, Robert R, et al. The dose response multicentre investigation on fluid assessment (DoReMIFA) in critically ill patients. Crit Care. 2016;20(1):196.

8 Neyra JA, Li X, Canepa-Escaro F, AdamsHuet B, Toto RD, Yee J, et al. Cumulative fluid balance and mortality in septic patients with or without acute kidney injury and chronic kidney disease. Crit Care Med. 2016; 44(10):1891-900.

9 Heung M, Wolfgram DF, Kommareddi M, $\mathrm{Hu}$ Y, Song PX, Ojo AO. Fluid overload at initiation of renal replacement therapy is associated with lack of renal recovery in patients with acute kidney injury. Nephrol Dial Transplant. 2012;27(3):956-61.
10 Fulop T, Pathak MB, Schmidt DW, Lengvarszky Z, Juncos JP, Lebrun CJ, et al. Volume-related weight gain and subsequent mortality in acute renal failure patients treated with continuous renal replacement therapy. ASAIO J. 2010;56(4):333-7.

11 Casas-Aparicio GA, León-Rodríguez I, Hernández-Zenteno RJ, Castillejos-López M, Alvarado-de la Barrera C, Ormsby CE, et al. Aggressive fluid accumulation is associated with acute kidney injury and mortality in a cohort of patients with severe pneumonia caused by influenza A H1N1 virus. PLoS One. 2018;13(2):e0192592.

12 Sakr Y, Rubatto Birri PN, Kotfis K, Nanchal R, Shah B, Kluge S, et al. Higher fluid balance increases the risk of death from sepsis: results from a large international audit. Crit Care Med. 2017;45(3):386-94.

13 Li Y, Wang J, Bai Z, Chen J, Wang X, Pan J, et al. Early fluid overload is associated with acute kidney injury and PICU mortality in critically ill children. Eur J Pediatr. 2016;175(1):39-48.

14 Balakumar V, Murugan R, Sileanu FE, Palevsky $\mathrm{P}$, Clermont G, Kellum JA. Both positive and negative fluid balance may be associated with reduced long-term survival in the critically ill. Crit Care Med. 2017;45(8):e749-57.

15 Myles PS, Bellomo R, Corcoran T, Forbes A, Peyton P, Story D, et al. Restrictive versus liberal fluid therapy for major abdominal surgery. N Engl J Med. 2018;378(24):2263-74.

16 Kuo G, Chen SW, Lee CC, Chen JJ, Fan PC, Wang SY, et al. Latent trajectories of fluid balance are associated with outcomes in cardiac and aortic surgery. Ann Thorac Surg. 2020 May;109(5):1343-9.

17 Yende S, Kellum JA, Talisa VB, Peck Palmer $\mathrm{OM}$, Chang $\mathrm{CH}$, Filbin MR, et al. Long-term host immune response trajectories among hospitalized patients with sepsis. JAMA Netw Open. 2019;2(8):e198686.

18 Jiang L, Zhu Y, Luo X, Wen Y, Du B, Wang $\mathrm{M}$, et al. Epidemiology of acute kidney injury in intensive care units in Beijing: the multicenter BAKIT study. BMC Nephrol. 2019; 20(1):468.
19 Knaus WA, Draper EA, Wagner DP, Zimmerman JE. APACHE II: a severity of disease classification system. Crit Care Med. 1985; 13(10):818-29.

20 Vincent JL, de Mendonca A, Cantraine F, Moreno R, Takala J, Suter PM, et al. Use of the SOFA score to assess the incidence of organ dysfunction/failure in intensive care units: results of a multicenter, prospective study. Crit Care Med. 1998;26(11):1793-800.

21 Le Gall JR, Lemeshow S, Saulnier F. A new simplified acute physiology score (SAPS II) based on a European/North American multicenter study. JAMA. 1993;270(24):2957-63.

22 Khwaja A. KDIGO clinical practice guidelines for acute kidney injury. Nephron Clin Pract. 2012;120(4):C179-84.

23 Levey AS, Coresh J, Balk E, Kausz AT, Levin A, Steffes MW, et al. National Kidney foundation practice guidelines for chronic kidney disease: evaluation, classification, and stratification. Ann Intern Med. 2003;139(2):13747.

24 Hoste EA, Clermont G, Kersten A, Venkataraman R, Angus DC, De Bacquer D, et al. RIFLE criteria for acute kidney injury are associated with hospital mortality in critically ill patients: a cohort analysis. Crit Care. 2006; 10(3):R73.

25 Ma YC, Zuo L, Chen JH, Luo Q, Yu XQ, Li Y, et al. Modified glomerular filtration rate estimating equation for Chinese patients with chronic kidney disease. J Am Soc Nephrol. 2006;17(10):2937-44.

26 Nagin DS, Odgers CL. Group-based trajectory modeling in clinical research. Annu Rev Clin Psychol. 2010;6:109-38.

27 Twisk J, Hoekstra T. Classifying developmental trajectories over time should be done with great caution: a comparison between methods. J Clin Epidemiol. 2012;65(10): 1078-87.

28 Nagin DS, Tremblay RE. Analyzing developmental trajectories of distinct but related behaviors: a group-based method. Psychol Methods. 2001;6(1):18-34. 
29 Schwandt A, Hermann JM, Rosenbauer J, Boettcher C, Dunstheimer D, Grulich-Henn $\mathrm{J}$, et al. Longitudinal trajectories of metabolic control from childhood to young adulthood in type 1 diabetes from a large German/Austrian registry: a group-based modeling approach. Diabetes Care. 2017;40(3):309-16.

30 Jones BL, Nagin DS. A note on a stata plugin for estimating group-based trajectory models. Sociol Methods Res. 2013;42(4):608-13.

31 Desquilbet L, Mariotti F. Dose-response analyses using restricted cubic spline functions in public health research. Stat Med. 2010;29(9): 1037-57.

32 Monnet X, Marik PE, Teboul JL. Prediction of fluid responsiveness: an update. Ann Intensive Care. 2016;6(1):111.

33 Rhodes A, Evans LE, Alhazzani W, Levy MM, Antonelli M, Ferrer R, et al. Surviving sepsis campaign: international guidelines for management of sepsis and septic shock: 2016. Intensive Care Med. 2017;43(3):304-77.

34 Loflin R, Winters ME. Fluid resuscitation in severe sepsis. Emerg Med Clin North Am. 2017;35(1):59-74.

35 Lee J, de Louw E, Niemi M, Nelson R, Mark RG, Celi LA, et al. Association between fluid balance and survival in critically ill patients. J Intern Med. 2015;277(4):468-77.
36 Zinter MS, Spicer AC, Liu KD, Orwoll BE, Alkhouli MF, Brakeman PR, et al. Positive cumulative fluid balance is associated with mortality in pediatric acute respiratory distress syndrome in the setting of acute kidney injury. Pediatr Crit Care Med. 2019 Apr;20(4): 323-31.

37 Alobaidi R, Morgan C, Basu RK, Stenson E, Featherstone R, Majumdar SR, et al. Association between fluid balance and outcomes in critically ill children: a systematic review and meta-analysis. JAMA Pediatr. 2018;172(3): 257-68.

38 McDermid RC, Raghunathan K, Romanovsky A, Shaw AD, Bagshaw SM. Controversies in fluid therapy: type, dose and toxicity. World J Crit Care Med. 2014;3(1):24-33.

39 Chapalain X, Vermeersch V, Egreteau PY, Prat G, Alavi Z, Vicaut E, et al. Association between fluid overload and SOFA score kinetics in septic shock patients: a retrospective multicenter study. J Intensive Care. 2019;7: 42.

40 Codes L, de Souza YG, D’Oliveira RAC, Bastos JLA, Bittencourt PL. Cumulative positive fluid balance is a risk factor for acute kidney injury and requirement for renal replacement therapy after liver transplantation. World J Transplant. 2018;8(2):44-51.
41 Raimundo M, Crichton S, Syed Y, Martin JR, Beale R, Treacher D, et al. Low systemic oxygen delivery and $\mathrm{BP}$ and risk of progression of early AKI. Clin J Am Soc Nephrol. 2015;10(8): 1340-9.

42 Vaewpanich J, Akcan-Arikan A, Coss-Bu JA, Kennedy CE, Starke JR, Thammasitboon S. Fluid overload and kidney injury score as a predictor for ventilator-associated events. Front Pediatr. 2019;7:204.

43 Kuttab HI, Lykins JD, Hughes MD, Wroblewski K, Keast EP, Kukoyi O, et al. Evaluation and predictors of fluid resuscitation in patients with severe sepsis and septic shock. Crit Care Med. 2019;47(11):1582-90.

44 Woodward CW, Lambert J, Ortiz-Soriano V, Li Y, Ruiz-Conejo M, Bissell BD, et al. Fluid overload associates with major adverse kidney events in critically ill patients with acute kidney injury requiring continuous renal replacement therapy. Crit Care Med. 2019; 47(9):e753-60.

45 Hammond DA, Lam SW, Rech MA, Smith MN, Westrick J, Trivedi AP, et al. Balanced crystalloids versus saline in critically ill adults: a systematic review and meta-analysis. Ann Pharmacother. 2020;54(1):5-13.

46 Barea-Mendoza J, Chico-Fernández M, Montejo-González JC. Balanced crystalloids versus saline in critically ill adults. N Engl J Med. 2018;378(20):1950-1. 